\title{
Das deutsche IMI
}

\section{Das Impact Message Inventory nach Kiesler und seine Circumplexstruktur}

\section{Das Impact Message Inventory (IMI) von Don Kiesler ist ein inter- personaler Fragebogen, der zur Beurteilung zwischenmenschlicher Eigenschaften durch Zweitpersonen eingesetzt wird. Ein besonderes Merkmal: Die beurteilende Person beschreibt die Wirkung der be- urteilten Person auf sich. Das IMI wird mit seiner Einbettung in den Interpersonalen Ansatz und seiner Entwicklung über mehrere Stufen der Verbesserung eingeführt. Es folgt eine Darstellung der Überset- zung und Evaluation der deutschen Version, anschließend wird der Zugang zum Fragebogen und zum Auswertungsschlüssel erklärt.}

\section{Einleitung}

\section{Der Interpersonale Ansatz und sein Einfluss}

"Interpersonal Theory of Psychiatry“ Hinter dem IMI (Impact Message Inventory) von Don Kiesler (Kiesler 1983) stecken zwei Ideen: Die erste geht auf Harry Stack Sullivan zurück (Sullivan 1953). Dieser hat Grundlagen des Interpersonalen Ansatzes entworfen und dem Verständnis von psychischen Störungen als Störungen der zwischenmenschlichen Beziehungen zugrunde gelegt. Sullivan veröffentlichte 1953 seine „Interpersonal Theory of Psychiatry“ (Sullivan 1953). Er stellte bei seiner Erklärung psychischer Probleme die aktuellen zwischenmenschlichen Beziehungen ganz in den Vordergrund. Dass ein zentrales Motiv aus seiner Sicht dabei die Angstreduktion ist, passt sehr gut auch zu einer modernen bindungstheoretischen Sicht zwischenmenschlicher Beziehungen (Strauß 2011). Sullivan wurde zur Kenntnis genommen und übte einen gewissen Einfluss aus. Allerding war der ursprüngliche Ansatz in weiten Teilen noch nicht besonders klar ausformuliert. Er wurde weiterentwickelt und ausformuliert, eine Reihe von Messmitteln wurden entwickelt (s. unten), und ein Therapieansatz - entwickelt insbesondere für Depressionen (Klerman et al. 1984) - wurde „Interpersonal Psychotherapy“ genannt.

Der CBASP-Ansatz Später verwendete ein Kollege Kieslers an der Virginia Commonwealth University, James McCullough, dies als wichtige Basis seines integrativen CBASP-Ansatzes (Cognitive Behavioral Analysis System of Psychotherapy) für die Behandlung chronischer Depressionen (Mc Cullough 2000). Dies hat gerade auch im deutschen Sprachraum zu einem starken Anstieg des Interesses am IMI geführt.

Die Attraktivität des Interpersonalen Ansatzes bestand und besteht u. a. darin, dass ein dritter, wissenschaftlich orientierter Weg in der Kontroverse zwischen psychodynamischen und behavioralen Ansätzen eröffnet wurde (Horowitz et al. 2006).

Der Interpersonale Ansatz war auch ein wichtiges Standbein für den Plan-Ansatz nach Grawe \& Caspar (Grawe 1908, Caspar 1989) mit seiner Betonung der Analyse aktueller Beziehungen.
Weiterentwicklung Vieles hat Sullivan nicht so klar formuliert. Es brauchte Interpretationen, Klärungsversuche und Weiterentwicklungen durch Kollegen wie Leary (Leary 1957), Carson (Carson 1969), Kiesler (Kiesler 1982), oder Horowitz et al. (Horowitz et al. 1988, Horowitz et al. 2006), um das eigentliche Potenzial des Ansatzes zu erschließen. Zu den besonderen Merkmalen des Interpersonalen Ansatzes gehört das Bemühen um eine Abbildung interpersonaler Stile in einem einfachen System. Leary hat das sogenannte Circumplex-Modell ausgearbeitet. Die Grundidee ist einfach: Die möglichen bzw. real beobachteten interpersonalen Eigenschaften in einem interpersonalen Raum anzuordnen. Dabei kommt er mit 2 Dimensionen aus, in denen jede interpersonale Position durch lediglich 2 Koordinaten beschreibbar wird.

Koordinatensystem aus 2 Achsen In verschiedenen Beiträgen etwas unterschiedlich formuliert, aber inhaltlich in etwa dasselbe meinend, werden 2 Achsen postuliert ( $\bullet$ Abb. 1 ):

- vertikale Achse: Control/Agency/Dominance, auf deutsch: Kontrolle

- horizontale Achse: Affiliation/Communion, auf deutsch meist auch Affiliation Elegant daran ist vor allem, dass jede Interpersonale Position durch 2 einfache Koordinaten ausgedrückt werden kann. Das Verhältnis der 2 Zahlen drückt dabei aus, in welcher Richtung sich die interpersonale Position einer Person - vom Nullpunkt aus gesehen - befindet. Die Höhe der Zahlen drückt aus, wie extrem die Position ist.

- Eine Position $(0,0)$ würde also bedeuten, dass die Person bzw. - je nachdem, was gemessen wird - ein Verhalten in einer bestimmten Situation in keinerlei Richtung auffällt. 
- Eine $(5,0)$ auf einer 5-stufigen Skala und bei Erwähnung der vertikalen Achse an erster Stelle würde bedeuten, dass die Person/das Verhalten extrem dominant und weder besonders freundlich noch hostil (feindselig) ist,

- eine $(2,2)$, dass sie / es deutlich erhöht dominant-freundlich ist,

- eine $(0,-5)$, dass sie/ es extrem hostil und dabei weder ausgeprägt submissiv noch dominant ist, usw.

Der SASB-Ansatz Über längere Zeit wurden in der Psychotherapieforschung einzelne Ansätze verfolgt, die auf interpersonaler Basis entwickelt wurden, wie der SASBAnsatz (Structural Analysis of Social Behavior, Benjamin 1974). Sie erwarben sich mit den daraus abgeleiteten Messmitteln über Therapieschul-Grenzen hinweg Aufmerksamkeit und Verdienste. So warb z. B. der bekannte psychoanalytisch orientierte Forscher Hans Strupp sehr stark für den SASBAnsatz. Auch bei der Entwicklung eines Verständnisses für Persönlichkeitsstörungen erwies sich SASB theoretisch und empirisch als sehr geeignet (Benjamin 1992). Dennoch blieb der Einfluss des interpersonalen Ansatzes über längere Zeit vergleichsweise bescheiden.

\section{Die Grundidee des IMI}

Beschreibung ist nie wirklich objektiv Die zweite, vom interpersonalen Kreismodell unabhängige Idee stammt von Kiesler (Kiesler 1978): Wenn eine Person interpersonale Eigenschaften einer zweiten Person beschreibt, tue sie das nicht wirklich aus einer Beobachterperspektive. Vielmehr stecke hinter der Beschreibung das, was die beschriebene Person bei der beschreibenden Person auslöst (Impact). Es wäre also direkter, wenn beschrieben würde, was ausgelöst wird, statt so zu tun, als würde distanziert ein Merkmal der Zielperson beschrieben.

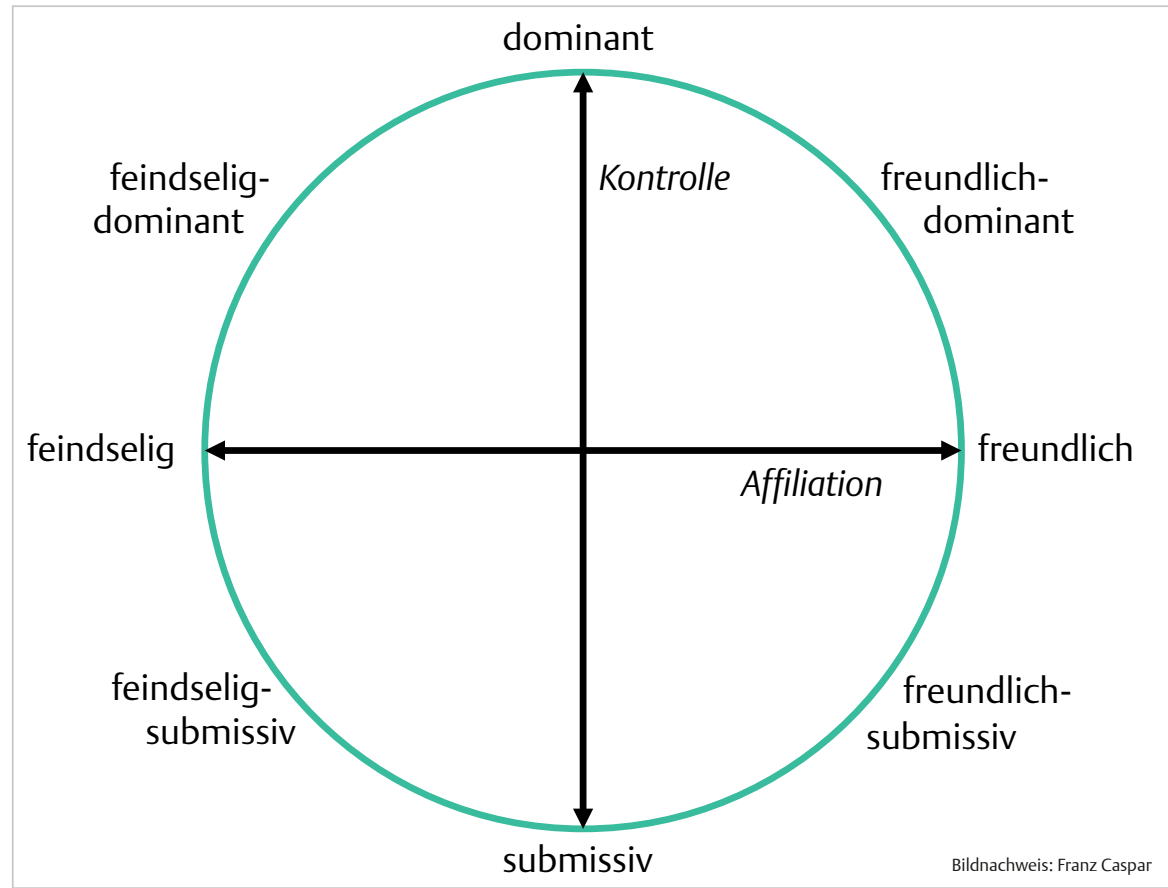

Abb. 1 Interpersonales Kreissystem, das durch 2 Dimensionen oder Achsen aufgespannt wird.

Deutlich wird dabei, dass wir es nicht mit einem Fremdbeurteilungsinstrument zu tun haben, bei dem der Betreuer neutral und objektiv wäre: Es gehen immer zwei Menschen mit ihren Eigenheiten in den Messprozess ein. Kiesler würde wohl sagen: Das ist bei jeder Fremdbeurteilung so, beim IMI ist es aber offensichtlicher und direkter.

\section{Übersetzung und Weiterentwicklung} Auf dieser Grundidee hat Kiesler das ursprüngliche IMI erarbeitet, das dann in mehreren Schritten weiterentwickelt wurde. Einige Zeit vor der verdienten Renaissance der Interpersonalen Ansätze wurde in der Berner Arbeitsgruppe eine erste Übersetzung entwickelt (Werner 1984, Caspar \& Werner 1985), evaluiert und umfangreich im klinischen Kontext eingesetzt. Sie erwies sich als wertvoll, aber noch weiter verbesserungswürdig. So entstand 1996 ein Versuch der Optimierung und Neuevaluation (Fingerle 1998). Diese Version wurde ebenfalls erfolgreich eingesetzt, es war al- lerdings fraglich, ob nun bezüglich der Faktorenstruktur schon das Optimum erreicht sei. Daraufhin wurde der Fragebogen erneut verbessert und evaluiert. Diese Evaluation wird zusammen mit inzwischen an Patienten gewonnenen Daten in diesem Beitrag vorgestellt.

Parallel haben auch Kiesler und Kollegen das englische IMI verbessert und neu evaluiert (Kiesler \& Schmidt 2006). Selbstverständlich stellt sich damit die Frage, ob die deutsche Version jeweils eine Übersetzung des neuesten amerikanischen Standes sein, oder unabhängig davon auf der Basis deutscher Vorgänger-Versionen weiterentwickelt werden soll. Vor dem Hintergrund der Verfügbarkeit des sehr klaren zweidimensionalen interpersonalen Modells einerseits, der ebenfalls sehr klaren Konstruktionsidee von Kiesler andererseits, zudem begründet in der Erfahrung, dass recht gut übersetzte Items in der 2-Faktoren-Struktur teils doch nicht dort zu liegen kommen, wo sie liegen sollten, wurde der zweite Weg gewählt und in diesem Artikel weitergeführt. 


\section{Das IMI im Kontext anderer interpersonaler Messmittel}

Das Inventar Interpersonaler Probleme Das IMI war im Englischen nicht gut evaluiert. Beim SASB-Ansatz hat seine Komplexität und die Schwierigkeit, seine verlässliche Anwendung zu erlernen, seine Verbreitung nicht gerade gefördert. So haben vermutlich verschiedene Faktoren zusammengespielt, die einem Boom des Interpersonalen Ansatzes eher entgegen standen.

Ein ganz zentraler Beitrag, der zum verstärkten Interesse an Interpersonalen Ansätzen seit Ende der 80er Jahre geführt hat, war die Entwicklung des Inventars Interpersonaler Probleme (IIP) durch Horowitz, Rosenberg, Baer, Ureno \& Villasenor (Horowitz et al. 1988). Es kam den Bedürfnissen der Psychotherapieforschung entgegen, auch den interpersonalen Bereich mit einem standardisierten Messmittel abzudecken. Das IIP wurde 1993 auf Deutsch veröffentlicht (Horowitz et al. 1993) und 1997 auch in eine konsensuell erarbeitete Liste empfohlener Messmittel aufgenommen (Horowitz et al. 1997).

Verschiedene Messmittel zeichnen sich dabei durch Besonderheiten aus:

- Das IMI ist ein Fremdbeurteilungsinstrument, das ohne Wertung / Problematisierung eine andere Person mittels eigener Erfahrungen beschreibt,

- das IIP eine Selbstbeurteilung interpersonaler Probleme,

- das SASB-System enthält auch eine Beurteilung von Introjekten, also den Umgang der Person mit sich selbst, usw.

Trotz dieser Unterschiedlichkeiten haben die Verfahren den Interpersonalen Ansatz und das zweidimensionale Koordinatensystem als gemeinsamen Kern, was Messungen damit auch vergleichbar und gegenseitig ergänzbar macht (Pincus et al. 2010).

Aufteilung anhand eines Kreises Eine andere Variation ist die Aufteilung des durch die 2 Dimensionen aufgespannten Kreises: Grundlegend sind die insgesamt 4 Pole der 2 Dimensionen. Die Quadranten, die zwischen ihnen liegen, können weiter zu Oktanten halbiert werden, und für jeden Oktanten kann man eine entsprechende Skala formulieren, beim IMI z. B.

- dominant,

- feindselig-dominant,

- feindselig,

- feindselig-submissiv,

- submissiv,

- freundlich-submissiv,

- freundlich und

- freundlich-dominant.

Weitere Aufteilungen zu 16, 32, und 64 Segmenten werden auch vorgeschlagen, aber seltener verwendet. Werden die Messwerte von den Skalen im Kreis herum eingetragen, ergibt sich ein Profil, aus dem man mit etwas Übung auf einen Blick die qualitative (Ausprägungen in welchen Bereichen) und quantitative (wie stark sind die Ausprägungen) Situation erfassen kann.

\section{Widersprüchliche Einschätzungen} möglich Weil interpersonale Messmittel wie der IMI mehr als 2 Skalen beinhalten, die durch voneinander unabhängige Items erfasst werden, ist es möglich, dass eine Person gleichzeitig freundlich-submissiv („rechts unten“ im interpersonalen Zirkel) wie auch feindselig-dominant (,links oben“ im interpersonalen Zirkel) eingeschätzt wird. Bei einem System mit 2 bipolaren Dimensionen könnte man ja erwarten, dass man nicht gleichzeitig an mehreren Stellen auf einer Dimension liegen kann. Deswegen muss man zwischen der Lokalisierung einer Person im interpersonalen Raum und der pragmatischen Darstellung der Ausprägungen auf einzelnen der 8 einpoligen interpersonalen Skalen unterscheiden.

- Eine Person hat nur einen Ort im interpersonalen Raum.

- Betrachtet man dagegen zusammen die 8 einpoligen Skalen, die im Kreis angeordnet sind, kann eine Person gleichzeitig mehrere Werte auf verschiedenen Skalen haben.

Situation und Interaktionspartner spielen eine Rolle Empirisch werden unterschiedliche Ausprägungen auf verschiedenen interpersonalen Skalen oft be- obachtet. Geht es um die Beurteilung einer Person, kann man sich vorstellen, dass in Abhängigkeit von der Situation und dem Interaktionspartner durchaus verschiedene interpersonale Positionen eingenommen werden können. Ein populäres Beispiel ist die „Radfahrer-Strategie“: „nach oben buckeln und nach unten trampeln“. Immer wieder trifft man auch Menschen, die am Arbeitsplatz freundlich-submissiv sind und den aufgestauten Ärger dann zuhause feindselig-dominant herauslassen. Wenn die Beziehung zu einer einzelnen Person oder gar ein Verhalten in einer bestimmten Situation auf gegensätzlichen Positionen landet, kann das daran liegen, dass implizit einerseits das Verhalten, andererseits vermutete Motive bzw. ausgelöste Gefühle der Einschätzung zugrunde gelegt werden.

Komplementaritäts-Konzept Zentral und vor allem auch praktisch sehr nützlich ist das Komplementaritäts-Konzept: Es geht davon aus, dass

- submissives Verhalten dominantes Verhalten einlädt und vice versa,

- freundliches freundliches und

- feindseliges feindseliges.

In Bezug auf die Kontroll-Dimension liegt das komplementäre Verhalten also gegenüber, in Bezug auf die Affiliation-Dimension auf derselben Seite.

Stabile Muster bilden sich dann, wenn die Positionen von zwei Interaktionspartnern komplementär sind. Ist das nicht der Fall, ergeben sich keine stabilen Muster, d.h., wenn man kann, geht man sich eher aus dem Weg oder interagiert möglichst wenig. Wenn man nicht ausweichen kann, ergeben sich spannungsreiche Muster.

Rolle des Therapeuten Es gibt aber auch Ausnahmen von der einfachen Regel. So passt es nicht zur Therapeutenrolle, dass ein Therapeut auf einen feindseligen Patienten feindselig reagiert. Er wird grundsätzlich darauf eingestellt sein, Patienten freundlich zu begegnen, und sich beherrschen, wenn 
dieser sich feindselig verhält. McCullough (2000) hat sehr schön herausgearbeitet, dass das nicht ohne Folgen bleibt: Innerlich geht ein solches Angehen gegen den komplementären Impuls nicht spurlos vorüber. Therapeuten entwickeln hinter ihrem freundlichen Verhalten hostile Gefühle, die sich dann doch bisweilen in hostilem Verhalten oder dem Wunsch, mit diesem Patienten möglichst nichts mehr zu tun zu haben, und oft in Therapieabbrüchen oder unguten Beziehungen äußern. McCullough nutzt das IMI intensiv, um hostiles, meist vordergründig submissives Problemverhalten von Patienten aufzuzeigen, Therapeuten den komplementären „Pull“ in eine dominante, eigentlich feindselig/distanzierende Richtung vor Augen zu führen und damit die Chance zu erhöhen, dass sie ihm widerstehen.

\section{IMI als Erfassungsinstrument und Untersuchungsobjekt}

\section{Evoking und impact messages}

Definitionen Kiesler definiert Persönlichkeit als ein relativ gleich bleibendes Muster von sich wiederholenden Evoking- und Impact-Messages (Kiesler 1983). EvokingMessages sind dabei zumeist verschlüsselte Botschaften des Senders, die neben einer Selbstdarstellung auch eine Aufforderung an den Empfänger enthalten, sich komplementär zu verhalten. Unter Impact-Message versteht Kiesler die Reaktion des Empfängers auf die Evoking-Message des Senders. Jedes Individuum entwickelt ein spezifisches Muster aus Evoking-Messages, welches beim Empfänger bestimmte ImpactMessages auslöst.

Da die Evoking-Messages zumeist unbewusst sind, können diese über Selbstbeurteilungsverfahren nicht erhoben werden. Um also etwas über die Persönlichkeit des Individuums erfahren zu können, müssen die Impact-Messages des Empfängers erfasst werden.
Umsetzung im IMI Im IMI wurde für jeden interpersonalen Oktanten eine Skala entwickelt, wobei jeweils

- die Kognitive Reaktion („ich habe den Eindruck, er möchte im Zentrum der Aufmerksamkeit stehen“),

- die Affektive Reaktion (,ich fühle mich herumkommandiert") und

- die Verhaltenstendenz/der Verhaltensimpuls („ich möchte mich schützen“) mit Items bedacht werden.

Das Original-IMI wie auch die deutschen Übersetzungen enthalten 64 Items, also 8 Items pro Skala, mit 4-stufigen Antworten von „stimmt gar nicht“ bis „stimmt genau“. Wie bereits erwähnt, war das ursprüngliche IMI im Englischen nicht gut evaluiert: Es wurden nur wenige Personen bewertet, und dabei handelte es sich nicht einmal um reale, sondern nur um beschriebene Personen. Bei der Übersetzung ins Deutsche bestand deshalb von Anfang an ein Interesse, eine bessere Evaluation - v.a. mit realen Personen - vorzunehmen.

\section{Das deutsche IMI}

Übersetzung und Anpassung der englischen Version Die Entwicklung eines Fragebogens in einer anderen Sprache ist nicht einfach eine Übersetzung. Das mag man an einer Verschlechterung der Kennwerte wie Interne Konsistenz oder Validität merken. Bei einem interpersonalen Fragebogen wird eine Veränderung der psychologischen Bedeutung eines Items besonders schnell deutlich, weil vom Konzept her klar ist, wo in der Kreisstruktur ein Item auch in der Zielsprache zu liegen kommen sollte. Die üblichen Schritte des Übersetzens und Rückübersetzens unter Einbeziehung eines muttersprachlichen Beurteilers wurden auch beim IMI unternommen (Werner 1984; Caspar \& Werner 1985). Hinzu kam eine Faktorenanalyse mit Messwerten an einer Stichprobe aus der Normalbevölkerung, bei der die Lage jedes Items in der Faktorenstruktur untersucht wurde. Für jedes Item wurde dann detailliert festgehalten, welche Verbesserungen oder Verschlechterungen einzelne Umformulierungen ergaben.
Bei einzelnen Items halfen alle Änderungen der Übersetzung nicht. Dann wurde ausgehend von der Vorgabe (um welchen Oktanten, welchen der 3 Aspekte - affektive oder kognitive Reaktion, Verhaltensimpuls - geht es?) eine Neuentwicklung versucht und wiederum evaluiert.

\section{Kulturspezifische Formulierungen} Man muss sich klarmachen, dass das Treffen der richtigen Position mit einer Formulierung ja nicht nur eine Sache der Sprache ist, sondern dass gerade bei interpersonalen Themen kulturelle Unterschiede eine große Rolle spielen. Ein bekanntes Beispiel ist die Bedeutung eines „date“ in der amerikanischen Kultur, die im deutschen Sprachraum keine wirkliche Entsprechung hat. Selbstverständlich gibt es auch keine einheitliche deutschsprachige Interaktionskultur: Stadt / Land, Alterskohorten, geografische Regionen etc. unterscheiden sich. In klinischen Stichproben werden im Allgemeinen viel mehr submissive als dominante Personen gefunden, usw. Dass dadurch bei einer Messung Ungenauigkeiten auftreten, ist aber keine Besonderheit Interpersonaler Messmittel oder des IMI: Wenn überhaupt ein Messmittel verwendet werden soll, das eine gewisse Allgemeinheit hat, müssen hier Unschärfen in Kauf genommen werden.

\section{Verwendung im klinischen Kontext}

Ziele Im klinischen Kontext wurde und wird der IMI vielfach eingesetzt. Unter anderem geht es darum,

- analog zu Veränderungen in Symptomatik / Beschwerden im Zusammenhang mit der Therapie auch Veränderungen im zwischenmenschlichen Verhalten zu erfassen,

- Fallkonzeptionen/Therapieplanungen auf vom klinischen Eindruck unabhängige Messungen interpersonaler Merkmale zu stützen,

- auf mögliche Komplikationen in der Therapiebeziehungen aufmerksam zu werden,

- auf schlechtere Voraussetzungen für Veränderungen in der Therapie generell 
aufmerksam zu werden (es gibt Hinweise, dass dominant-hostile Patienten sich in ihrer Symptomatik besonders schwer, freundlich-submissive Patienten besonders leicht verändern [Schauenburg et al. 2000; Filak et al. 1986]), und

- Studierende und Therapeuten im Beobachten zwischenmenschlichen Verhaltens und im Deuten eigener Reaktionen auf Patienten anzuleiten.

Erkenntnisse Bei allen Vorbehalten gegenüber Schwächen, die in den einzelnen Versionen verblieben sind, kann doch festgehalten werden, dass in verschiedenen Untersuchungen immer wieder nützliche Erkenntnisse gewonnen wurden, auf die hier detaillierter einzugehen aber kein Raum besteht (u.a. Grosse Holtforth et al. 2012).

Generell findet man im Prä-Post-Vergleich (vor- vs. nach Therapie) in interpersonalen Messmitteln - nicht nur im IMI - kleinere Veränderungen als etwa in Beschwerdelisten. Das macht durchaus Sinn, weil ja nur bei einem Teil der Patienten der interpersonale Stil verändert werden soll - und wenn, dann ist die erwünschte Veränderung meist auf einzelne Aspekte des zwischenmenschlichen Verhaltens beschränkt. Auch scheint der interpersonale Stil im Allgemeinen gegenüber Veränderungen etwas träger zu sein als konkretere Problemverhaltensweisen.

Eine gesteigerte Aufmerksamkeit hat der IMI in jüngster Zeit durch die Anwendung bei der Beurteilung des interpersonalen Stils chronisch depressiver Patienten und darauf aufbauende Reflexionen zur Komplementarität im Zusammenhang mit McCulloughs auch im deutschen Sprachraum populär gewordenem CBASP-Ansatz erfahren (McCullough 2013).

\section{Untersuchung}

\section{Aktualisierungen des englischen und des deutschen IMI}

Das amerikanische IMI wurde zum IMI-C weiterentwickelt, das neu 56 Items, also 7 pro Skala enthält. Normen finden sich bei
Kiesler und Auerbach (Kiesler \& Auerbach 2003), Angaben zur Testgüte bei Schmidt, Wagner und Kiesler (Schmidt et al. 1999). Man findet bei insgesamt 1109 Personen allgemein

- eine Bestätigung der 2-Faktorenstruktur mit 69\% Varianzaufklärung und

- eine akzeptable bis gute interne Konsistenz (Cronbach's Alpha zwischen 0,69 und 0,89 ).

Etwas früher erfolgten durch Fingerle (Fingerle 1998) beim deutschen IMI - nach wie vor auf der ursprünglichen amerikanischen IMI beruhend - neue Anstrengungen zur Verbesserung namentlich der Faktorenstruktur, die zunächst an einer hier nicht näher beschriebenen kleinen Stichprobe von $n=34$ aus der Normalbevölkerung überprüft wurde.

\section{Anlage der Untersuchung und Stichproben}

Einseitige Antworttendenzen eingedämmt In einer neuen Version des Fragebogens sollten v.a. vermutete Antworttendenzen in eine unproblematischsympathische Richtung eingedämmt werden. Um dieses Problem zu lösen, wurden Veränderungen der Formulierungen einzelner Items vorgenommen. Dabei ging es v.a. darum, die Varianz bei Items mit geringer Varianz zu erhöhen. So wurde z. B. bei Item 1 „fühle ich mich herumkommandiert“ (Original: feel bossed around“) die geringe Varianz in der Werner-Version darauf zurückgeführt, dass viele Decoder wohl ungern zugeben, sich herumkommandieren zu lassen. Das neue „sie möchte mir sagen, was ich tun oder lassen soll“ zeigte wie erwartet eine bessere Varianz.

Bei der Instruktion wurde die Aufforderung hinzugefügt, spontan zu antworten, und es wird ausgedrückt, „dass jeder Mensch positive und negative Eigenschaften hat, die Persönlichkeit erst ausmachen“, um eine Tendenz in Richtung sozialer Erwünschtheit etwas abzuschwächen. Diese neueste Version wurde in einer Normalbevölkerungs- und in einer klinischen Stichprobe untersucht.
Stichprobe Normalbevölkerung In der Normalbevölkerungs-Stichprobe wurde eine „Sympathievariation“ eingefügt, d.h. ein Teil der Personen wurde aufgefordert, ihnen sympathische, ein Teil, ihnen unsympathische Personen zu beschreiben. Damit sollte letzteren leichter gemacht werden, im Fragebogen auch Negatives auszudrücken. Eine Neuevaluation erfolgte an einer Stichprobe von $n=184$. Von den Probanden wohnten 152 Personen in Deutschland und 32 Personen in der Schweiz. 64\% waren Frauen, das Durchschnittsalter lag bei 43 Jahren (SD = 18,5; Altersspanne: 17-88 Jahre).

Die zu beurteilende Person (Encoder) wurde von den Decodern unter „gut bekannten Personen“ jeweils selbst ausgewählt. 94 Personen wählten eine Frau, 90 Personen einen Mann. Bezüglich des Berufstandes besuchten 4 Decoder die Schule, 36 absolvierten ein Studium, 66 waren berufstätig, 18 arbeiteten als Hausfrau und 50 befanden sich in Pensionierung (10 Personen ohne Angabe). Betreffend des Bildungsniveaus hatten 51 Decoder die Haupt- bzw. Primarschule besucht, 44 die Real- bzw. Sekundarschule und 95 das Gymnasium (2 Personen ohne Angabe). Insgesamt werden damit keine größeren Verzerrungen gegenüber der Normalbevölkerung deutlich.

Klinische Stichprobe Weiter wurde an der Psychotherapeutischen Ambulanz des Instituts für Psychologie, Universität Bern eine Überprüfung des IMI an einer unabhängigen klinischen Prüfstichprobe vorgenommen. Dabei handelt es sich um 464 Patienten, die von 2000-2008 ambulant behandelt wurden und von nahen Bezugspersonen beurteilt wurden. Das IMI wird an der Ambulanz zu Beginn jeder Therapie standardmäßig erhoben.

Das Alter der eingeschätzten Patienten lag zwischen 18 und 80 Jahren (Mittel: 35,98; SD: 12,2$) .56 \%$ der Patienten waren Frauen, 58,4\% der Stichprobe waren ledige Personen. Nach DSM-IV - diagnostiziert mit dem strukturierten klinischen Interview (SKID) - war bei 149 Patienten (32,1\%) als Hauptdiagnose eine Angststörung diagnos- 
tiziert worden. Affektive Störungen wurden bei 141 (30,4\%), Anpassungsstörungen bei 44 (9,5\%), Essstörungen bei 15 (3,2\%) und somatoforme Störungen bei 14 (3,0\%) diagnostiziert.

Die Decoder waren nahe Bezugspersonen: in 2/3 der Fälle Partnerinnen und Partner (im Alter durchschnittlich etwa entsprechend dem der Patienten), das restliche Drittel etwa gleich verteilt Familienmitglieder (Eltern, erwachsene Kinder) und nahestehende Freunde.

\section{Auswertung}

Neben der Berechnung von Mittelwerten, Standardabweichungen und der internen Konsistenz (Cronbach's Alpha) der theoretisch postulierten Skalen wurden explorative Hauptkomponentenanalysen durchgeführt. Auf Basis der rotierten Komponentenmatrix wurde anschließend die Circumplexstruktur für die theoretisch zu erwartende und empirisch gefundene 2-faktorielle Lösung (2 Dimensionen des interpersonalen Zirkels) überprüft. Hierfür wurde der Winkel der Faktorladungen der einzelnen Items und der 8 postulierten Skalen im Koordinatensystem des interpersonalen Zirkels berechnet und mit den theoretisch zu erwartenden Winkeln verglichen (s.a. Schmidt et al. 1999).

\section{Ergebnisse}

\section{Mittelwert, Standardabweichung und interne Konsistenz der Skalen}

Mittelwerte, Standardabweichungen und die interne Konsistenz (Cronbach's Alpha) sind in 0 Tab. 1 dargestellt. Cronbach‘s Alpha bewegt sich in der Normalstichprobe zwischen 0,74 (dominant) und 0,97 (freundlich). Auch bei der klinischen Stichprobe erweist sich die interne Konsistenz als akzeptabel bis gut: zwischen 0,68 (freundlichsubmissiv) und 0,86 (freundlich; freundlichdominant).

Tab. 1 Mittelwert, Standardabweichung und interne Konsistenz (Cronbach's Alpha) der einzelnen Skalen bei einer Normal- und einer klinischen Stichprobe

\begin{tabular}{|l|c|c|c|c|c|c|}
\hline & \multicolumn{3}{|c|}{ Bevölkerungsstichprobe $(\mathbf{n}=184)$} & \multicolumn{2}{l|}{ klinische Stichprobe $(\mathbf{n = 4 6 4 )}$} \\
\hline Skala & $\begin{array}{l}\text { Mittel- } \\
\text { wert }\end{array}$ & $\begin{array}{l}\text { Standard- } \\
\text { abweichung }\end{array}$ & $\begin{array}{l}\text { Cronbach's } \\
\text { Alpha }\end{array}$ & $\begin{array}{l}\text { Mittel- } \\
\text { wert }\end{array}$ & $\begin{array}{l}\text { Standard- } \\
\text { abweichung }\end{array}$ & $\begin{array}{l}\text { Cronbach's } \\
\text { Alpha }\end{array}$ \\
\hline freundlich-dominant & 2,72 & 0,95 & 0,9 & 2,58 & 0,63 & 0,86 \\
\hline dominant & 2,76 & 0,94 & 0,74 & 2,34 & 0,55 & 0,75 \\
\hline feindselig-dominant & 2,49 & 1,09 & 0,93 & 2,06 & 0,62 & 0,82 \\
\hline feindselig & 2,30 & 1,16 & 0,95 & 1,72 & 0,51 & 0,79 \\
\hline feindselig-submissiv & 2,04 & 0,98 & 0,87 & 2,32 & 0,64 & 0,80 \\
\hline submissiv & 2,02 & 0,95 & 0,84 & 2,44 & 0,65 & 0,81 \\
\hline freundlich submissiv & 2,29 & 0,99 & 0,85 & 2,88 & 0,44 & 0,68 \\
\hline freundlich & 2,76 & 1,16 & 0,97 & 3,36 & 0,55 & 0,86 \\
\hline
\end{tabular}

\section{Hauptkomponentenanalyse und Analyse der Circumplex-Struktur}

2 Faktoren Vorausgeschickt sei, dass ein größeres $\mathrm{n}$ bei der nicht-klinischen Stichprobe als Basis für eine Faktoranalyse wünschenswert wäre. Die Ergebnisse der explorativen Hauptkomponentenanalyse bei der Bevölkerungsstichprobe legen die erwartete Zweifaktorenlösung nahe:

- Die beiden ersten Faktoren erklären zusammen $82,4 \%$ der Varianz,

- wobei der Eigenwert des dritten Faktors $<1$ liegt.

Ein etwas anderes Bild zeigt sich bei der klinischen Stichprobe: Hier weisen insgesamt 12 Komponenten einen Eigenwert > 1 auf.

- Die beiden ersten Faktoren erklären zusammen 36\% der Varianz (1. Faktor: $22 \%$; 2 . Faktor: $12 \%$ ),

- wobei die restlichen 10 Komponenten alle je $<4 \%$ der Varianz erklären,

was zumindest gemäß dem Scree-Kriterium (Cattell 1966) auch für die Extraktion zweier Faktoren sprechen würde.

Möglicherweise ist die eindeutigere Reduktion auf 2 Faktoren in der Normalstichprobe darauf zurückzuführen, dass in dieser Stichprobe gezielt auch unsympathische Personen beschrieben wurden. Dies hat möglicherweise zu einer größeren Varianz in den Daten und zu einer klareren Struktur als in der klinischen Stichprobe geführt.

Circumplexstruktur Zur Klärung der Frage, ob die Items und Skalen eine Cir- cumplexstruktur aufweisen, wurde auf der Basis der rotierten Komponentenmatrix der zweifaktoriellen Lösung der Winkel der Faktorladungen der einzelnen Items und der 8 postulierten Skalen im Koordinatensystem des interpersonalen Zirkels berechnet und mit den theoretisch zu erwartenden Winkeln verglichen. Gemäß dem Circumplexmodell ist $\mathrm{z}$. $\mathrm{B}$.

- für die Skala „dominant“ ein Winkel von $90^{\circ}$,

- für „freundlich-dominant“ ein Winkel von $45^{\circ}$ und

- für freundlich ein Winkel von $0^{\circ}$ bzw. $360^{\circ}$ zu erwarten (Schmidt et al. 1999).

In 0 Tab. 2 und 3 sind die zu erwartenden und empirisch gefundenen Winkel der 8 Skalen des IMI-R sowie die maximale Abweichung der Items der einzelnen Skalen und die Anzahl der Items mit einer Abweichung $>22,5^{\circ}$ dargestellt. Eine Abweichung $>22.5 \%$ bedeutet, dass ein Item auf den beiden interpersonellen Faktoren so lädt, dass es näher zu einem anderen als dem theoretisch zu erwartenden Oktanten zu liegen kommt.

Erläuterungen Aus $\bullet$ Tab. 3 wird ersichtlich, dass sich in den Daten auf Skalenniveau tatsächlich eine Circumplexstruktur abbildet. Die durchschnittliche Abweichung vom theoretisch zu erwarteten Winkel liegt bei 9, $1^{\circ}$, wobei die Abweichung maximal 13,7 beträgt (Skala „feindselig-submissiv). Auf Item-Ebene laden 14 der 64 Items näher zu einem jeweils benachbarten statt dem the- 
Tab. 2 Bevölkerungsstichprobe

\begin{tabular}{|c|c|c|c|c|c|}
\hline Skala & $\begin{array}{l}\text { erwarteter } \\
\text { Winkel }\end{array}$ & $\begin{array}{l}\text { gefundener } \\
\text { Winkel }\end{array}$ & $\begin{array}{l}\text { Diskre- } \\
\text { panz }\end{array}$ & $\begin{array}{l}\text { maximale } \\
\text { Abweichung } \\
\text { der Items } \\
\text { einer Skala }\end{array}$ & $\begin{array}{l}\text { Anzahl Items } \\
\text { mit Abwei- } \\
\text { chung }>22,5^{\circ}\end{array}$ \\
\hline freundlich-dominant & $45^{\circ}$ & $37,5^{\circ}$ & $-7,5^{\circ}$ & $26,8^{\circ}$ & 2 \\
\hline dominant & $90^{\circ}$ & $98,6^{\circ}$ & $+8,6^{\circ}$ & $23,5^{\circ}$ & 1 \\
\hline feindselig-dominant & $135^{\circ}$ & $137,3^{\circ}$ & $+2,3^{\circ}$ & $27,3^{\circ}$ & 1 \\
\hline feindselig & $180^{\circ}$ & $180,4^{\circ}$ & $+0,4^{\circ}$ & $8,6^{\circ}$ & 0 \\
\hline feindselig-submissiv & $225^{\circ}$ & $215,6^{\circ}$ & $+9,4^{\circ}$ & $20,9^{\circ}$ & 0 \\
\hline submissiv & $270^{\circ}$ & $274,3^{\circ}$ & $+4,3^{\circ}$ & $25,1^{\circ}$ & 2 \\
\hline freundlich submissiv & $315^{\circ}$ & $315,6^{\circ}$ & $+0,6^{\circ}$ & $8,6^{\circ}$ & 0 \\
\hline freundlich & $360^{\circ}$ & $360,5^{\circ}$ & $+0,5^{\circ}$ & $3,6^{\circ}$ & 0 \\
\hline \multicolumn{3}{|c|}{ Durchschnittliche Diskrepanz } & $4,2^{\circ}$ & & \\
\hline \multicolumn{5}{|c|}{ Total Items mit Abweichung $>22,5^{\circ}$} & 6 \\
\hline
\end{tabular}

\section{Tab. 3 Klinische Stichprobe}

\begin{tabular}{|c|c|c|c|c|c|}
\hline Skala & $\begin{array}{l}\text { erwarteter } \\
\text { Winkel }\end{array}$ & $\begin{array}{l}\text { gefundener } \\
\text { Winkel }\end{array}$ & $\begin{array}{l}\text { Diskre- } \\
\text { panz }\end{array}$ & $\begin{array}{l}\text { maximale } \\
\text { Abweichung } \\
\text { der Items } \\
\text { einer Skala }\end{array}$ & $\begin{array}{l}\text { Anzahl Items } \\
\text { mit Abwei- } \\
\text { chung }>22,5^{\circ}\end{array}$ \\
\hline freundlich-dominant & $45^{\circ}$ & $55,5^{\circ}$ & $+10,5^{\circ}$ & $15,2^{\circ}$ & 0 \\
\hline dominant & $90^{\circ}$ & $79,7^{\circ}$ & $-10,3^{\circ}$ & $43,8^{\circ}$ & 4 \\
\hline feindselig-dominant & $135^{\circ}$ & $147,1^{\circ}$ & $+12,1^{\circ}$ & $70,5^{\circ}$ & 1 \\
\hline feindselig & $180^{\circ}$ & $182,4^{\circ}$ & $+2,4^{\circ}$ & $21,7^{\circ}$ & 0 \\
\hline feindselig-submissiv & $225^{\circ}$ & $238,7^{\circ}$ & $+13,7^{\circ}$ & $33,7^{\circ}$ & 2 \\
\hline submissiv & $270^{\circ}$ & $256,6^{\circ}$ & $-13,4^{\circ}$ & $25,5^{\circ}$ & 3 \\
\hline freundlich submissiv & $315^{\circ}$ & $304,5^{\circ}$ & $-10,5^{\circ}$ & $48,4^{\circ}$ & 4 \\
\hline freundlich & $360^{\circ}$ & $364,5^{\circ}$ & $+4,5^{\circ}$ & $11,1^{\circ}$ & 0 \\
\hline \multicolumn{3}{|c|}{ Durchschnittliche Diskrepanz } & $9,1^{\circ}$ & & \\
\hline \multicolumn{5}{|c|}{ Total Items mit Abweichung $>22,5^{\circ}$} & 14 \\
\hline
\end{tabular}

oretisch erwarteten Oktanten (Abweichung $>22,5^{\circ}$ ).

Bei den hiervon betroffenen Skalen (z. B. „dominant“) gleicht sich diese Abweichung auf Skalenebene insofern aus, als z.B. jeweils 2 Items in die entgegengesetzte Richtung abweichen. Dadurch liegt der Mittelwert dort, wo er theoretisch liegen sollte. Die Abweichungen erklären aber die für die betroffenen Skalen etwas geringer ausgefallen internen Konsistenzen ( $\bullet$ Tab. 2).

Bei einem Item der Skala „feindselig-dominant" konnte eine Abweichung $>67,5^{\circ}$ gefunden werden (Item 59: „dass ich Rücksicht nehme“), was bedeutet, dass es am stärksten auf dem um einen Oktanten entfernten „feindselig-submissiv“ lädt. Dieses
Item sollte bei einer Weiterentwicklung des Fragebogens sicher besonders kritisch betrachtet werden.

Insgesamt kann bei einem Messmittel wie dem IMI nicht mit einer über alle denkbaren relevanten Stichproben hinweg völlig stabilen Struktur gerechnet werden. Jede neue Untersuchung könnte Anlass zu kleineren Veränderungen bei den Items geben, die sich jedoch bei jeder weiteren neuen Stichprobe als obsolet oder gar nachteilig erweisen kann.

Mit diesem Vorbehalt kann jedoch das IMI als genügend abgesichert betrachtet werden, um in der aktuellen, bei uns und informell bei vielen anderen seit Jahren eingesetzten Form zur Verfügung gestellt zu werden.

\section{Das aktuelle IMI in deutscher Sprache}

Lizenzgebühren Der Entwickler des amerikanischen IMI, D. Kiesler hat bereits unsere erste Übersetzung ins Deutsche begrüßt, gefördert und auch in Publikationen darauf hingewiesen. Von Lizenzgebühren war damals nie die Rede. Nach seinem Tod wurden die Rechte strenger verwertet, und der amerikanische IMI kostet dort Lizenzgebühren. Die Autoren dieses Beitrages sehen das als hinderlich für die verbreitete Verwendung, namentlich die Forschung, finanzieren eine Abgeltung der Gebühren gegenüber der amerikanischen Verwertungsgesellschaft selber, und stellen das IMI mit dieser Publikation zur kostenlosen Benutzung zur Verfügung. Benutzer, die das IMI in großem Umfang verwenden möchten, sind gebeten, sich mit dem Erstautor in Verbindung zu setzen und einen bescheidenen freiwilligen Beitrag zu den Lizenzgebühren zu entrichten. Es gibt eine männliche und eine weibliche Form.

Die aktuellen Items unterliegen dem Copyright, ihre Eigenbenutzung ist aber kostenlos möglich. Den Fragebogen in männlicher und weiblicher Person finden Sie auf www. mindgarden.com/IMIGerman. Weitere Informationen, auch zur Auswertung, erhalten Sie auf der Homepage des Erstautors www.imi.psy.unibe.ch.

Das IMI kann ohne Weiteres nach dem Auswertungsschlüssel auf der MindGardenHomepage ausgewertet werden. Von Fangmeier wurde zusätzlich ein kostenloses Auswertungsprogramm entwickelt, das auch eine grafische Darstellung produziert. Zugang ist über die Homepage des Erstautors möglich. 


\section{Diskussion, Zusammenfassung und Ausblick}

Verhalten ist verschieden deutbar Bei aller Eleganz stoßen die Interpersonalen Messansätze auch an Grenzen. Ein einfaches Beispiel: Eine aufopfernde, sich an Anforderungen in der Familie anpassende, immer zur Verfügung stehende Mutter mag bei oberflächlicher Betrachtung ihres Verhaltens im interpersonalen Kreis reliabel rechts unten platziert werden. Bei genauerer Betrachtung übt sie aber durch ein solches Verhalten auch ein hohes Maß an (oft unoffener) Macht aus. In einer Plananalyse (Caspar 2007) lässt sich das gut als instrumentelle Strategie auf verschiedenen hierarchischen Ebenen darstellen: Das Verhalten kann als „passt sich an auftretende Forderungen und Aufgaben aufopfernd an“ oder ähnlich bezeichnet werden. In der Hierarchie (per definitionem weitgehend unbewusster) Pläne könnte das dann so aussehen wie in $\bullet$ Abb. 2 dargestellt.

Differenzierte Erfassung schwierig Die Bezüge lassen sich (hier nur als Illustration, nicht als abschließende Analyse gemeint) also in Ansätzen, die sich auch als interpersonal verstehen, durchaus differenziert verstehen und darstellen. Der einfache interpersonale Messansatz stößt hier dagegen an seine Grenzen. Im IMI mögen sich die übergeordneten Motive durchaus darstellen, indem ein Impact des Sich-KontrolliertFühlens angegeben wird; im SASB mag (als Hinweis für Kenner des Ansatzes) eine sog. Komplex-Kodierung erfolgen und einen Hinweis auf etwas kompliziertere Verhältnisse geben. Richtig differenziert erfassen lassen diese sich aber nach unserem Kenntnisstand nicht.

Inkonsistente Befunde zur Komplementarität Eine neuere Veröffentlichung von Horowitz et al. (2006) reflektiert auch etwas genauer und empirisch fundiert die Komplementarität. Als Gegenpol zu Communion (was in anderen Beiträgen der freundlichen Position auf der Affiliation-

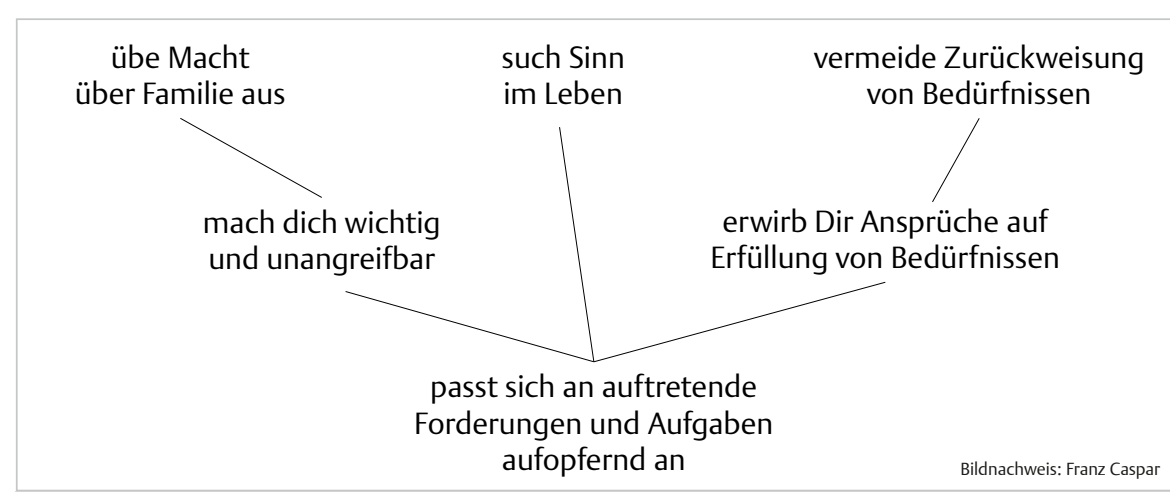

Abb. 2 Verhalten mit unoffenen übergeordneten Motiven (Verhalten entsprechend den Konventionen der Plananalyse im Indikativ, Pläne/Motive im an sich selbst gerichteten Imperativ formuliert).

Achse entspricht) wird nicht Hostility, sondern Indifference postuliert. Ganz im Einklang mit der Position der Plananalyse und ihrer komplementären bzw. motivorientierten Beziehungsgestaltung wird als komplementär nicht mehr definiert, was bei oberflächlichem Rating auf der Verhaltensebene interpersonal komplementär ist, sondern was die Motive des Gegenübers befriedigt. Damit werden auch interindividuelle Unterschiede betont: Verhalten, das für eine Person komplementär wäre, muss dies für eine andere Person nicht sein.

Mit diesen Veränderungen und Erweiterungen reagieren die Autoren auf inkonsistente Befunde zur Komplementarität, wonach insbesondere auf hostiles Verhalten oft nicht wie erwartet hostil reagiert wird. Sie fordern insbesondere, das „evokes complementary behavior“ durch ,invites“ zu ersetzen: Der Impuls ist also, wie ja auch bei McCullough beschrieben, da, wird aber nicht unbedingt in Verhalten umgesetzt.

Diese „Komplikationen“ werden hier bewusst referiert, um deutlich zu machen, dass die Grenzen einfacher Messungen mit dem IMI und anderen Messmitteln bekannt sind. Die Empfehlung, diese Messmittel zu verwenden, beruht also nicht auf Ignoranz gegenüber den beschriebenen Problemen, sondern auf der Überzeugung, dass die Messmittel einschl. IMI dennoch einen hohen praktischen Nutzen haben.

\section{Schlussfolgerungen und Fazit für die Praxis}

Brauchbares Instrument für die Praxis Der IMI ist ein wichtiges interpersonales Messmittel - das einfachste für Fremdbeurteilungen. Es kann im klinischen und nichtklinischen Kontext eingesetzt werden, wobei je nach Stichprobe mit leicht unterschiedlichen Lagen der einzelnen Items zu rechnen ist, die jedoch auf die Skalenwerte im allgemeinen keinen großen Einfluss haben sollten. Gerade weil die Nachfrage u.a. im Rahmen des CBASP-Ansatzes gestiegen ist, scheint es an der Zeit, die Phase der inoffiziellen Weitergabe des Fragebogens und Auswertungsschlüssels zu beenden und den Fragebogen unter Regelung lizenzrechtlicher Angelegenheiten auch offiziell zu veröffentlichen.

Komplexere Validitätsüberprüfung Die Überprüfung der Validität ist etwas komplexer als bei klassischen Fremdbeurteilungen, weil in die Messung deutlich immer die Varianz von 2 Personen eingeht. Ob hier wirklich ein Unterschied besteht, oder ob der Varianzbeitrag des Beurteilers hier lediglich offener erkennbar ist, ist diskutierbar: So oder so wäre es wünschbar, eine Untersuchung der Validität nach verschiedenen Kriterien zu ergänzen. 
Feedback erwünscht Wir werden die Untersuchung an weiteren Stichproben fortführen und bitten andere Forschergruppen um Mitteilung ihrer Erfahrungen und Ergebnisse. Daraus mögen sich auch kleinere Veränderungen der Items ergeben. Nach einer langen Serie von Verbesserungen an mehreren Stichproben kann das IMI in seiner jetzigen Form guten Gewissens zur Verfügung gestellt und verwendet werden.

\section{Kein Widerspruch zur neurobiologi-} schen Sicht In jüngster Zeit hat sicherlich die neurobiologische Sicht psychischer Störungen eine starke Aufmerksamkeit in Forschung und in der Öffentlichkeit auf sich gezogen und stellt insofern eine harte Konkurrenz für die interpersonale Sicht dar. Die beiden Perspektiven inhaltlich gegeneinander auszuspielen erscheint aber über das Ziel hinaus geschossen. Eine Vielzahl von Autoren sucht die auch von Grawe (Grawe 2004) geforderte gegenseitige Ergänzung. $\mathrm{Zu}$ diesen gehören interessanterweise gerade solche, die den interpersonal fundierten Therapieansatz verfolgen und sich gleichzeitig intensiv mit neurobiologischen Grundlagen beschäftigen (van Orden et al. 2010; Walter et al. 2013).

Wir danken M. Grosse Holtforth und J. Brodbeck für wertvolle Hinweise.

\section{Interessenkonflikt}

Der korrespondierende Autor gibt an, dass kein Interessenkonflikt besteht.

Beitrag online zu finden unter http://dx.doi.org/10.1055/s-0042-105981

\section{Hinweis zum Copyright}

Impact Message Inventory German translation, Copyright (c) 2006, 2016 by Donald J. Kiesler

All rights reserved in all media. Published by Mind Garden,

Inc. www.mindgarden.com.

German translation of the IMI by Franz Caspar
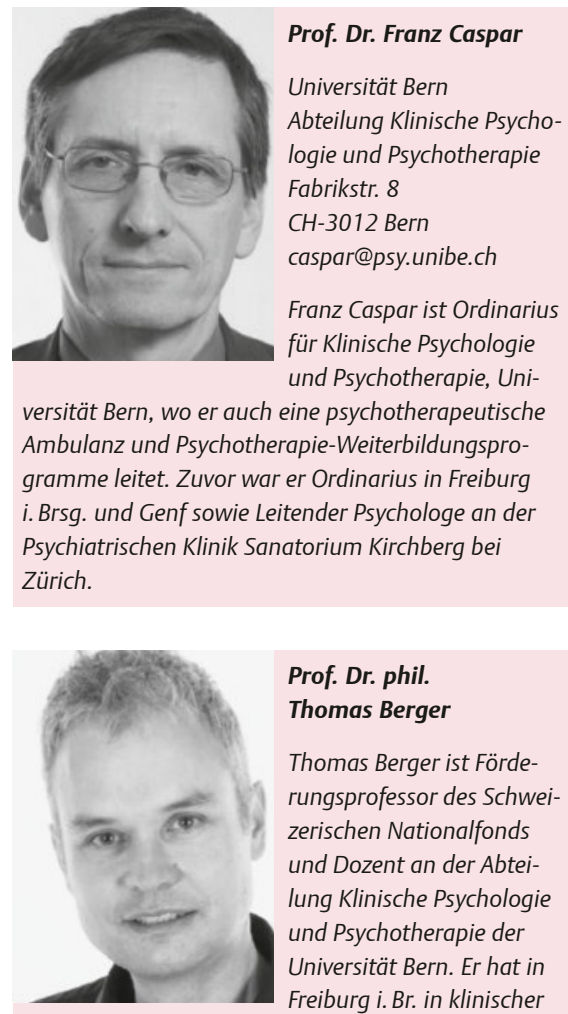

\section{Prof. Dr. phil. \\ Thomas Berger}

Thomas Berger ist Förderungsprofessor des Schweizerischen Nationalfonds und Dozent an der Abteilung Klinische Psychologie und Psychotherapie der Universität Bern. Er hat in Freiburg i. Br. in klinischer Psychologie promoviert und nach verschiedenen Tätigkeiten als Psychotherapeut (u. a. Sanatorium Kilchberg, ZH), einem Postdoc an den Universitäten Genf und Linköping (Schweden) in Bern habilitiert.

Dipl.-Psych. Helga Fingerle

Helga Fingerle hat ihren Beitrag zur Entwicklung des IMI-Fragebogens als Diplomandin am Psychologischen Institut in Tübingen geleistet.

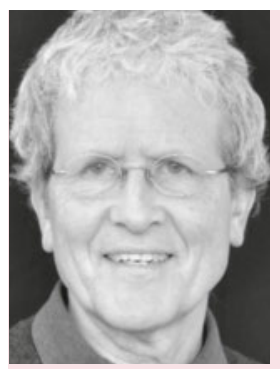

MA Martin Werner Martin Werner, geb. 1946, MA in Psychologie und Public Health, war nach dem Psychologiestudium in Bern am Institut Sozialund Präventivmedizin Bern in der arbeitsplatzbezogenen Gesundheitsforschung und in der Erforschung des HIV-relevanten Gesundheitsverhaltens tätig und anschließend am Bundesamt für Gesundheit als Wissensmanager in der HIV-Prävention. Seit der Berentung widmet er sich der seelsorgerisch-psychologischen Beratung. 
Literatur

Benjamin LS. An interpersonal approach to the diagnosis of borderline personality disorder. In: Clarkin JF, Marziali E, Monroe-Blum H, Hrsg. Borderline personality disorder. Clinical and empirical perspectives. New York: Guilford; 1992: 161-195

Benjamin LS. Structural analysis of social behavior. Psychol Rev 1974; 81: 392-425

Carson RC. Interaction concepts of personality. Chicago: Aldine; 1969

Caspar F, Werner M. The „IMI“ (Impact Message Inventory by Kiesler). Some general com-ments on measuring interpersonal relations, and the translation/validation of an instrument. In: Huber W, Hrsg. Progress in psychotherapy research. Selected Papers from the 2nd European Conference on Psychotherapy Research. Presses Universitaires: Louvain-la-Neuve; 1985: 496-511

Caspar F. Differenzierte und kreative Anstöße. Einige kasuistisch illustrierte Überlegungen zu Beziehung und Deutung in der Plananalyse. In: Reinelt T, Datler W, Hrsg. Beziehung und Deutung im psychotherapeutischen Prozess. Berlin: Springer; 1989: 144-160

Caspar F. Plan Analysis. In: Eells T, Hrsg. Handbook of psychotherapeutic case formulations. 2. Aufl. New York: Guilford; 2007: 251-289

Cattell RB. The scree test for the number of factors. Multivar Behav Res 1966; 1: 245-276

Filak J, Abeles N, Norquist S. Clients' pretherapy interpersonal attitudes and psychotherapy outcome. Prof Psychol-Res Pr 1986; 17: 217-222

Fingerle H. Der Impact Message Inventory. Deutsche Neukonstruktion [Diplomarbeit] Tübingen: Psychologisches Institut der Universität Tübingen; 1998

Grawe K. Die diagnostisch-therapeutische Funktion der Gruppeninteraktion in verhaltenstherapeutischen Gruppen. In: Grawe K, Hrsg. Verhaltenstherapie in Gruppen. München: Urban \& Schwarzenberg; 1980: 88-223

Grawe K. Psychological therapy. Seattle and Toronto: Hogrefe; 2004

Grosse Holtforth M, Altenstein D, Ansell E et al. Impact messages of depressed outpatients as perceived by their significant others: profiles, therapeutic change, and relationship to outcome. J Clin Psychol 2012; 68: 319-333

Horowitz LM, Lambert M], Strupp HH, Hrsg. Measuring patient change in mood, anxiety, and personality disorders: Toward a core battery. Washington, DC: American Psychological Association Press; 1997

Horowitz LM, Rosenberg S, Baer A et al. Inventory of interpersonal problems: psychometric properties and clinical applications. J Consult Clin Psych 1988; 57: 599-606

Horowitz LM, Strauß B, Kordy H. Inventar zur Erfassung interpersonaler Probleme. Deutsche Version (IIP-D). Weinheim: Beltz; 1993

Horowitz LM, Wilson KR, Turan B et al. How interpersonal motives clarify the meaning of interpersonal behavior: A revised circumplex model. Pers Soc Psychol Rev 2006; 10 : 67-86

Kiesler DJ, Auerbach SM. Integrating measurement of control and affiliation in studies of physician-patient interaction: The interpersonal circumplex. Soc Sci Med 2003; 57: 1707-1722

Kiesler DJ, Schmidt JA. Manual for the Impact Message Inventory-Circumplex (IMI-C). Menlo Park, CA: Mind Garden, Inc.; 2006

Kiesler DJ. A communication analysis of relationship in psychotherapy. Invited paper, read at the University of Minnesota Conference on Psychotherapy and Behavioral Intervention, Minneapolis, MN; 1978

Kiesler DJ. Interpersonal theory for personality and psychotherapy. In: Anchin JC, Kiesler DJ, Hrsg. Handbook of interper- sonal psychotherapy. Elmsford, NY: Pergamon; 1982: 274-295

Kiesler DJ. The 1982 Interpersonal Circle: A taxonomy for complementarity in human transactions. Psychol Rev 1983; 90: 185-214

Klerman GL, Weissman MM, Rounsaville B et al. Interpersonal psychotherapy of depression. New York: Basic Books; 1984

Leary T. Interpersonal diagnosis of personality. New York: Ronald; 1957

McCullough J. Einführung und state-of-the-art von CBASP. In: Belz M, Caspar F, Schramm E, Hrsg. Therapieren mit CBASP- München: Elsevier; 2013: 3-24

McCullough JP. Treatment for chronic depression. Cognitive Behavioral Analysis System of Psychotherapy. New York: Guilford Press; 2000

Pincus AL, Lukowitsky, MR, Wright AGC. The interpersonal nexus of personality and psychopathology. New York: Guilford Press; 2010

Schauenburg H, Kuda M, Sammet I et al. The influence of interpersonal problems and symptom severity on the duration and outcome of short-term psychodynamic psychotherapy. Psychother Res 2000; 10: 133-146

Schmidt JA, Wagner CC, Kiesler DJ. Psychometric and circumplex Properties of the octant scale Impact Message Inventory (IMI-C): A structural evaluation. J Couns Psychol 1999; 46: 325-334

Strauß B. Ergebnisse der klinischen Bindungsforschung mit Bedeutung für die Psychotherapie. Psychother Psych Med 2011; 61

Sullivan HS. The interpersonal theory of psychiatry. New York: Norton Press; 1953

van Orden K, Witte T, Cukrowicz K et al. The interpersonal theory of suicide. Psychol Rev 2010; 117: 575-600

Walter H, Schnell K, Klein P. Zur systemischen Neurowissenschaft der chronischen Depression. In: Belz M, Caspar F, Schramm E, Hrsg. Therapieren mit CBASP. München: Elsevier; 2013: 35-50

Werner M. Das Impact Message Inventory: Ein interpersonaler Persönlichkeitsfragebogen von Donald J. Kiesler (1976). Übersetzung aus dem Amerikanischen und erste Validierungen [Unpublished doctoral dissertation] Bern, Switzerland: University of Bern, $1984 \mathrm{w}$ 